\title{
On the claimed privacy of EC-RAC III *
}

\author{
Junfeng Fan, Jens Hermans ${ }^{\star \star}$, and Frederik Vercauteren ${ }^{\star \star \star}$ \\ Department of Electrical Engineering - COSIC \\ K.U.Leuven and IBBT \\ Kasteelpark Arenberg 10, B-3001 Leuven-Heverlee, Belgium \\ firstname.lastname@esat.kuleuven.be
}

\begin{abstract}
In this paper we show how to break the most recent version of EC-RAC with respect to privacy. We show that both the ID-Transfer and ID\&PWD-Transfer schemes from EC-RAC do not provide the claimed privacy levels by using a man-in-the-middle attack. The existence of these attacks voids the presented privacy proofs for EC-RAC.
\end{abstract}

Keywords: RFID, Protocols, EC-RAC, Privacy

\section{Introduction}

Radio Frequency Identification (RFID) is a technology that has great potential. It can be used in supply chains, access control, product authentication and so on. The study on RFID has mainly two branches: design of RFID-specific protocols and implementation of security components. The former focuses on design and analysis of cryptographic schemes that can meet various requirements in terms of security and privacy. The latter focuses on low-cost and secure implementations of cryptographic primitives such as hash functions and Public Key Cryptography (PKC).

The EC-RAC (ECDLP Based Randomized Access Control) protocol is a cryptographic protocol designed for RFID systems. It was designed to offer anonymity, which is not offered by conventional ECDLP based protocols such as the Schnorr [6] and the Okamoto [5] protocol. It was also carefully designed to "minimize the computation workload of a tag" [3]. The first version of the EC-RAC protocol [3] was broken in [7] and [1], while the second version of EC-RAC [4] was broken in [8]. In this paper,

* This work was supported in part by K.U. Leuven-BOF $(\mathrm{OT} / 06 / 40)$, by the IAP Programme P6/26 BCRYPT of the Belgian State (Belgian Science Policy), by FWO project G.0300.07, by the European Commission through the ICT programme under contract ICT-2007-216676 ECRYPT II.

$\star \star$ Research assistant, sponsored by the Fund for Scientific Research - Flanders (FWO).

${ }^{\star \star \star}$ Postdoctoral Fellow of the Fund for Scientific Research - Flanders (FWO). 
we examine the third version of EC-RAC [2] (EC-RAC III) and we show that it does not provide the claimed privacy properties.

The ID\&Pwd-Transfer protocols (protocol 2,3) are broken by a (wide) man-in-the-middle attack, and a tag can be traced by the attacker. Since our attacks on the ID\&Pwd-Transfer scheme do not require access to the tag's secrets, not even wide-weak privacy is provided by the protocols. Narrow-weak privacy might be provided by these protocols, but no formal proof for this is included. Also the ID-transfer protocol does not provide the claimed wide-strong privacy. An attacker that knows the identity of a certain tag, can always identify this tag using a man-in-the-middle attack. The highest privacy levels that could be provided by the IDTransfer scheme are narrow-strong privacy or wide-destructive, although no formal proof for this exists.

The remainder of the paper is structured as follows: in Section 2 we introduce the different versions of EC-RAC in detail and discuss the vulnerabilities of EC-RAC I and EC-RAC II. Section 3 introduces the privacy model of Vaudenay, which is used throughout this paper. In Section 4 we present our attacks on the various schemes of EC-RAC III and discuss the impact on the claimed privacy properties of the protocol.

\section{The EC-RAC protocols}

The basic setup considered in this paper is a world consisting of several tags and a single reader (or multiple connected to a central server). The reader/server is assumed trusted and the goal of the protocols is to authenticate the tag to the reader and, at the same time, protect the identity of the tag. Intuitively, it should be impossible for an adversary to impersonate a tag and it should be impossible for the adversary to derive any information on the identity of tags involved.

\section{$2.1 \quad$ EC-RAC I/II and related attacks}

The first version of the EC-RAC protocol was proposed in [3]. EC-RAC consists of several sub-protocols: ID-transfer, Pwd-Transfer and server authentication. The ID-transfer protocol allows the tag to identify itself to the server, the Pwd-Transfer protocol allows the tag to authenticate to the server. The two can be combined into the Id\&Pwd-Transfer protocol. Figure 1 shows the ID\&Pwd-Transfer protocol of EC-RAC I. Upper case symbols denote elliptic curve points, lower case symbols denote scalars.

This scheme was broken in [7] and [1], which show that a tag could be traced by an attacker using a quality-time attack [7]. If an attacker runs 
the protocol twice with the same $r_{2}$, collecting $\left\{v, T_{1}\right\}$ and $\left\{v^{\prime}, T_{1}^{\prime}\right\}$, she can then derive

$$
\left(v-v^{\prime}\right)^{-1}\left(T_{1}-T_{1}^{\prime}\right)=x_{1}^{-1} P
$$

which is a unique attribute of a tag. This unique attribute can then be used to identify the tag.

Fig. 1. ID\&Pwd-Transfer protocol from EC-RAC I [3]

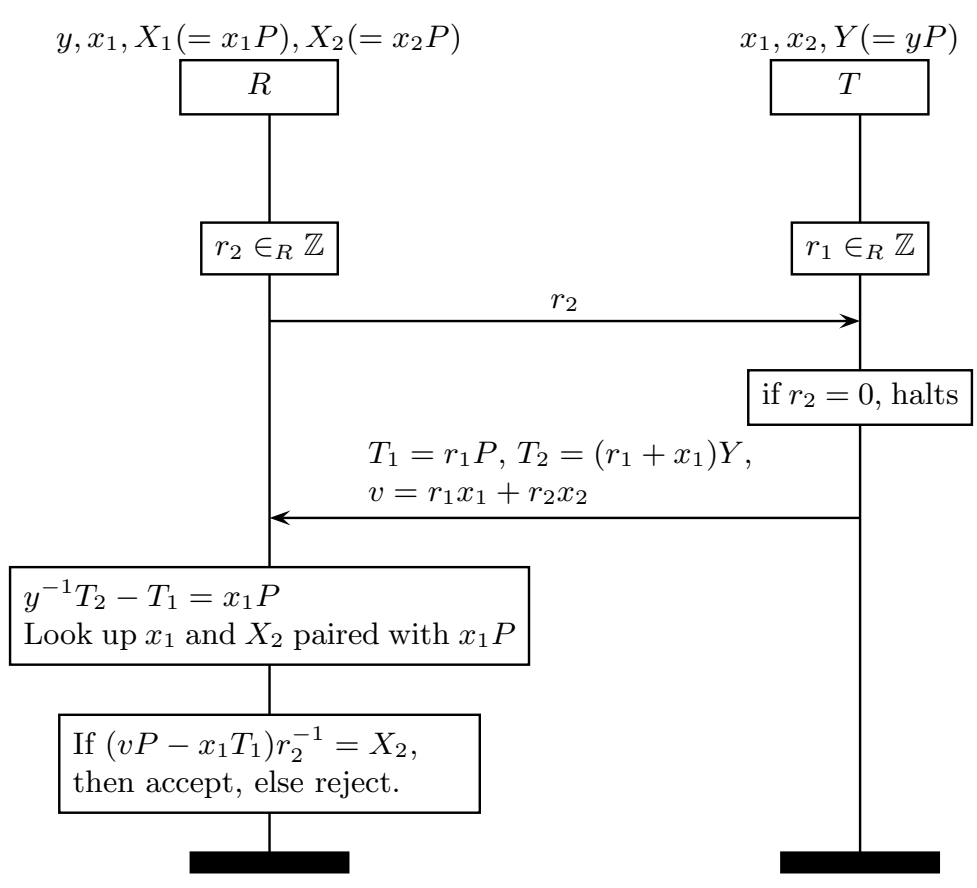

EC-RAC II [4] introduced three different sub-protocols: ID-transfer, Pwd-Transfer and server authentication. These sub-protocols were combined into several protocols. Figure 2 shows the ID transfer protocol.

EC-RAC II was broken in [8]. The ID-transfer scheme was broken with respect to untraceability using a man-in-the-middle attack, in which the attacker uses a previous, valid execution of the protocol to modify the 
Fig. 2. ID Transfer protocol from EC-RAC II [4]

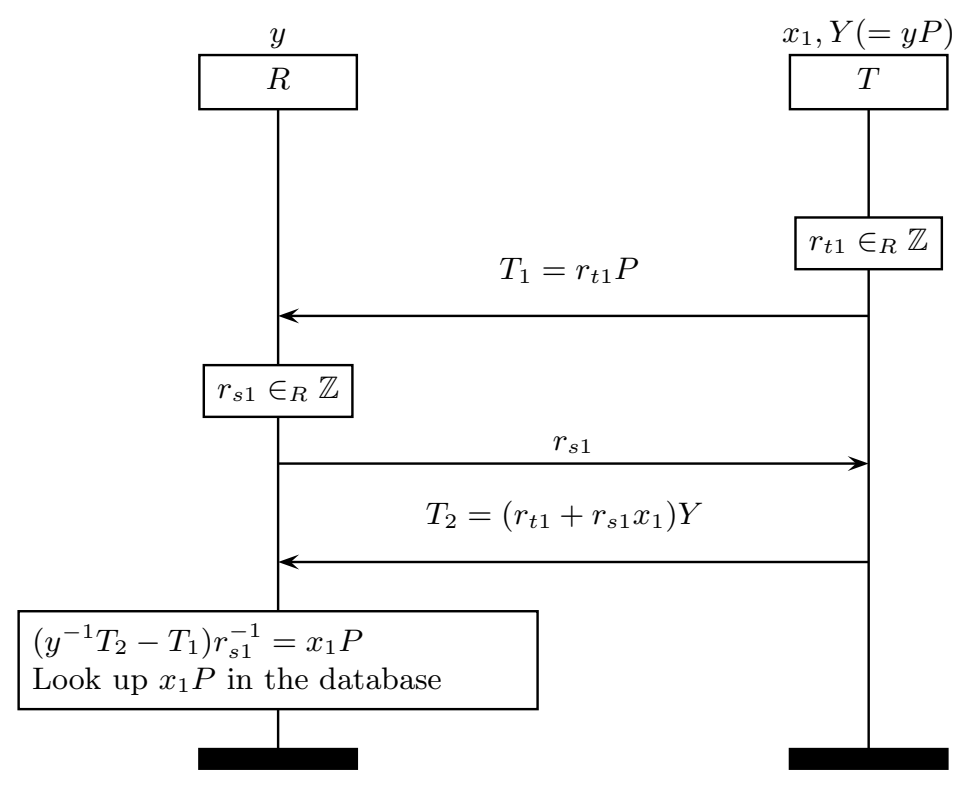

communication. If the reader accepts the modified values, the attacker can identify the previously eavesdropped tag.

One of the fundamental problems is that protocols, which in isolation are secure and/or untraceable, are not necessarily secure and/or privacy preserving when combined. The ID\&Pwd-Transfer protocols were broken with respect to tag-to-server authentication, allowing the attacker to impersonate a tag. The main cause of this attack is the reuse of the same randomness for both the ID- and Pwd-Transfer sub-protocol.

\subsection{EC-RAC III}

In [2] Lee, Batina, Singelée and Verbauwhede present an improved version of EC-RAC. The paper [2] claims that the ID-transfer protocol (protocol 1 from [2]) and the ID\&Pwd-Transfer protocol (protocol 3 from [2]) provide wide-strong privacy (see Section 3 for definition).

Let $P$ be a generator of the elliptic curve group. Every tag has two private-public key pairs $x_{1}, X_{1}=x_{1} P$ and $x_{2}, X_{2}=x_{2} P$. In this case $x_{1}$ 
serves as the identity of the tag and is also known by the reader. The reader has a private-public key pair $y, Y=y P$.

Figure 3 shows the ID-transfer protocol from [2]. This protocol should identify the tag as $x_{1}$ in a secure and wide-strong privacy preserving way. The main difference with the previous versions of the protocol is the introduction of the non-linearity $\dot{r}_{s}=x\left(r_{s} P\right)$, with $x(\cdot)$ the x-coordinate function for an elliptic curve point.

Fig. 3. ID-transfer protocol (Protocol 1) from [2]

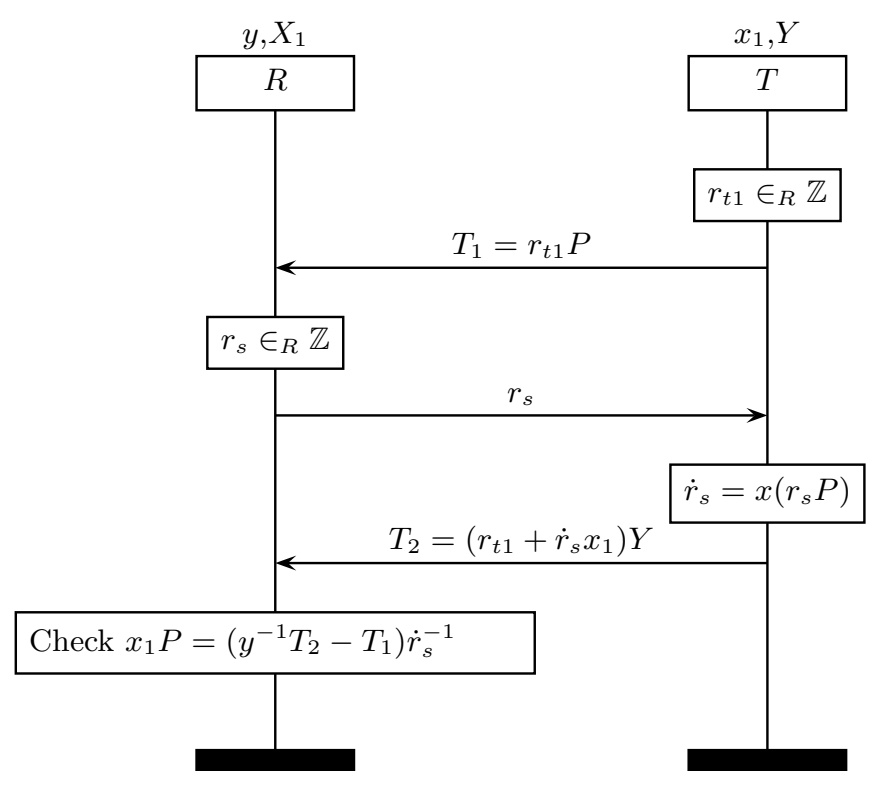

Figure 4 shows the ID\&Pwd-Transfer protocol from [2]. In addition to the reader identifying the tag correctly as $x_{1}$, it also authenticates the tag using the public-private key pair $x_{2}, X_{2}=x_{2} P$. (Note that the secret $x_{1}$ is known to both the tag and the reader and cannot be used for authentication.) 
Fig. 4. ID\&Pwd-Transfer protocol (Protocol 3) from [2]

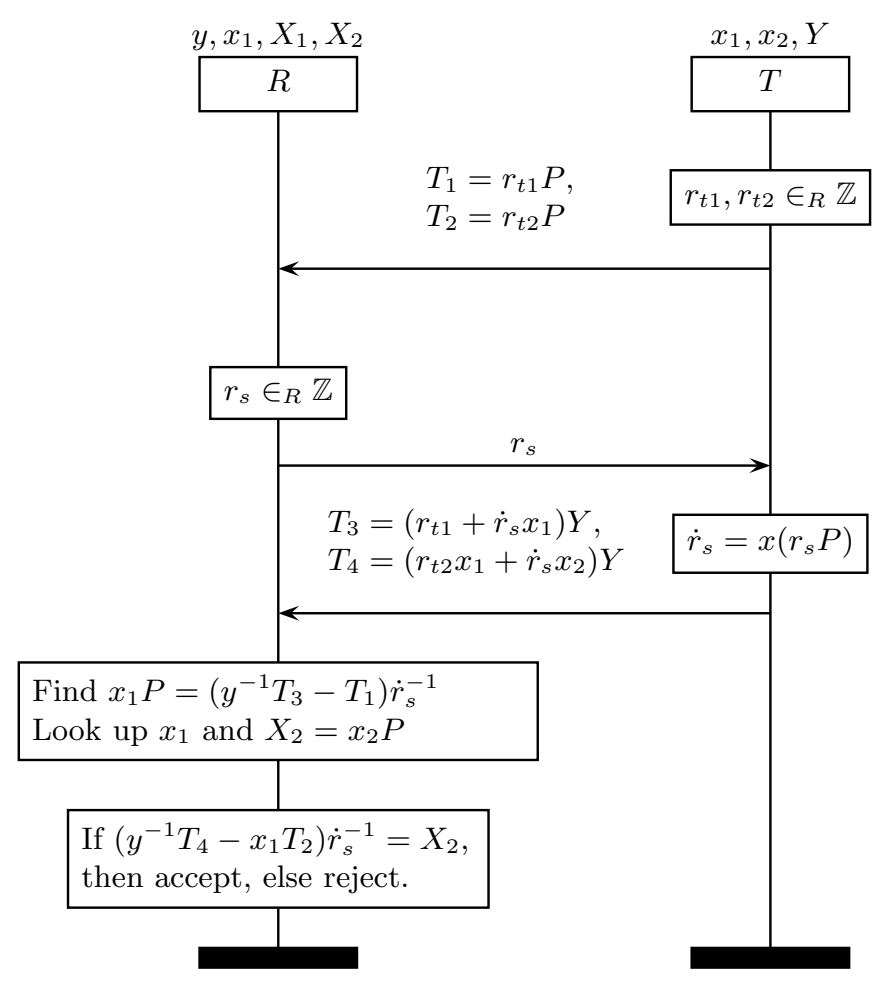

\section{Privacy Models}

Throughout this paper we use the privacy model from Vaudenay [9]. This model describes several oracles available to the attacker. For a complete list we refer to the original paper. Basically the attacker has the ability to perform a man-in-the-middle attack on any tag that is within its vincinity: it can influence all communication between tag and reader. The attacker also gets the result of the authentication of a tag, i.e. whether the reader accepts the tag or not. The attacker can also draw (at random) and free tags, moving them in and out of its range. During all of these interactions the attacker has to use a virtual identity to refer to the tags in its vincinity, i.e. it does not need to know the real identity to interact with a chosen 
tag. Finally the attacker can corrupt tags, reading out the entire internal state of the tag.

A strong attacker is allowed to use all the oracles available. A destructive attacker cannot use a tag anymore after it has been corrupted, i.e. corruption destroys the tag. In case of a forward attacker, the attacker can only do other corruptions after the first corruption. No protocol interactions are allowed after the first corrupt. A weak attacker does not have the ability to corrupt tags.

Orthogonal to these four attacker classes there is the notion of wide and narrow attackers. A wide attacker has access to the result of the verification by the server while a narrow attacker does not.

Definition 1. (Simplified version of Definition 6 from [9]) Privacy - A protocol is called $P$-private, with $P$ an adversary class from above (strong, destructive,...), if all adversaries belonging to the class $P$ are trivial.

Intuitively, an adversary is called trivial if it produces the same output, even when all protocol oracles are blinded (i.e. the attacker does not 'use' the communication captured during the protocol run to determine its output). Since the attacks presented in this paper allow tracing of tags, they clearly violate the privacy property, because the output of the attacker depends on information from the protocol runs that the attacker executes. As such, we do not require any detailed elements of the privacy definition used by Vaudenay.

The equations below show the most important relations between the privacy notions above:

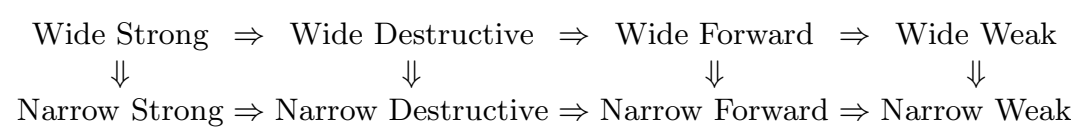

In this case $A \Rightarrow B$ means that if the protocol is A-private it implies that the protocol is B-private. It should be obvious that a protocol that is e.g. Wide Strong private will also belong to all other privacy classes above, that only allow weaker adversaries.

Besides privacy the protocol should also offer authentication of the tag. We refer to this property as the security of the protocol.

Definition 2. (Simplified version of Definition 4 from [9]) Security We consider any adversary in the class strong. The adversary wins if the reader identifies an uncorrupted legitimate tag, but the tag and the reader did not have a matching conversation. The RFID Scheme is called secure if the success probability of any such adversary is negligible. 


\section{Attacks on the protocols}

The main flaw in the ID\&Pwd-Transfer scheme is the fact that the "hash" of the challenge, i.e. $\dot{r}_{s}$ does not mask all of the secret keys $x_{1}$ and $x_{2}$. Indeed, in the response $T_{4}$, the $x_{1}$ part is only masked by the randomness $r_{t 2}$.

\subsection{First attack}

The first attack exploits the fact that it is possible to force $\dot{r}_{s}$ to become 0 . Indeed, note that the protocol does not verify whether $r_{s}$ is a multiple of the order of $P$. As such, it is possible for an attacker impersonating a reader to send $r_{s}=k \cdot \operatorname{ord}(P)$ to the tag, who will then compute $\dot{r}_{s}=x\left(r_{s} P\right)=0$ and therefore return $T_{3}=r_{t 1} Y$ and $T_{4}=r_{t 2} x_{1} Y$. Using the messages $\left(T_{1}=r_{t 1} P, T_{2}=r_{t 2} P, T_{3}=r_{t 1} Y, T_{4}=r_{t 2} x_{1} Y\right)$, it is then possible to mount a man-in-the-middle attack on a second communication to test whether the same tag from the first run is present or not. This attack is described in Figure 5 where the tag's secret keys are now denoted by $x_{1}^{\prime}$ and $x_{2}^{\prime}$.

The adversary adds $T_{1}$ and $T_{2}$ to the messages $T_{1}^{\prime}$ and $T_{2}^{\prime}$ obtained from the unknown tag and forwards these to the reader. The reader responds with a nonce $r_{s}^{\prime}$, which the attacker simply forwards to the tag. The tag responds with valid messages $T_{3}^{\prime}$ and $T_{4}^{\prime}$ which the attacker uses to obtain $T_{3}^{\prime \prime}=T_{3}^{\prime}+T_{3}$ and $T_{4}^{\prime \prime}=T_{4}^{\prime}+T_{4}$ and sends these to the reader. The reader then computes

$$
\left(y^{-1} T_{3}^{\prime \prime}-T_{1}^{\prime \prime}\right) \dot{r}_{s}^{\prime-1}=\left(r_{t 1}+r_{t 1}^{\prime}+\dot{r}_{s}^{\prime} x_{1}^{\prime}-r_{t 1}-r_{t 1}^{\prime}\right) \dot{r}_{s}^{\prime-1} P=x_{1}^{\prime} P,
$$

and looks up $x_{1}^{\prime}$ and $X_{2}^{\prime}=x_{2}^{\prime} P$. Note that this step always verifies. The reader then tests whether $\left(y^{-1} T_{4}^{\prime \prime}-x_{1}^{\prime} T_{2}^{\prime \prime}\right) \dot{r}_{s}^{\prime-1}=X_{2}^{\prime}$, which is equivalent with

$$
\left(r_{t 2}^{\prime} x_{1}^{\prime}+\dot{r}_{s}^{\prime} x_{2}^{\prime}+r_{t 2} x_{1}-x_{1}^{\prime}\left(r_{t 2}^{\prime}+r_{t 2}\right)\right) \dot{r}_{s}^{\prime-1} P=x_{2}^{\prime} P .
$$

The test will succeed if and only if $x_{1}=x_{1}^{\prime}$, i.e. if the tag is the same as the one from the first run.

\subsection{Second attack}

The second attack even works when the tag adds an extra verification that $\dot{r}_{s} \neq 0$. Note that the first attack worked because the attacker obtained $\left(T_{1}=r_{t 1} P, T_{2}=r_{t 2} P, T_{3}=r_{t 1} Y, T_{4}=r_{t 2} x_{1} Y\right)$, so it suffices to explain how such a tuple can be obtained when the tag verifies whether $\dot{r}_{s} \neq 0$. 
Fig. 5. Man-in-the-middle attack on protocols 2 and 3

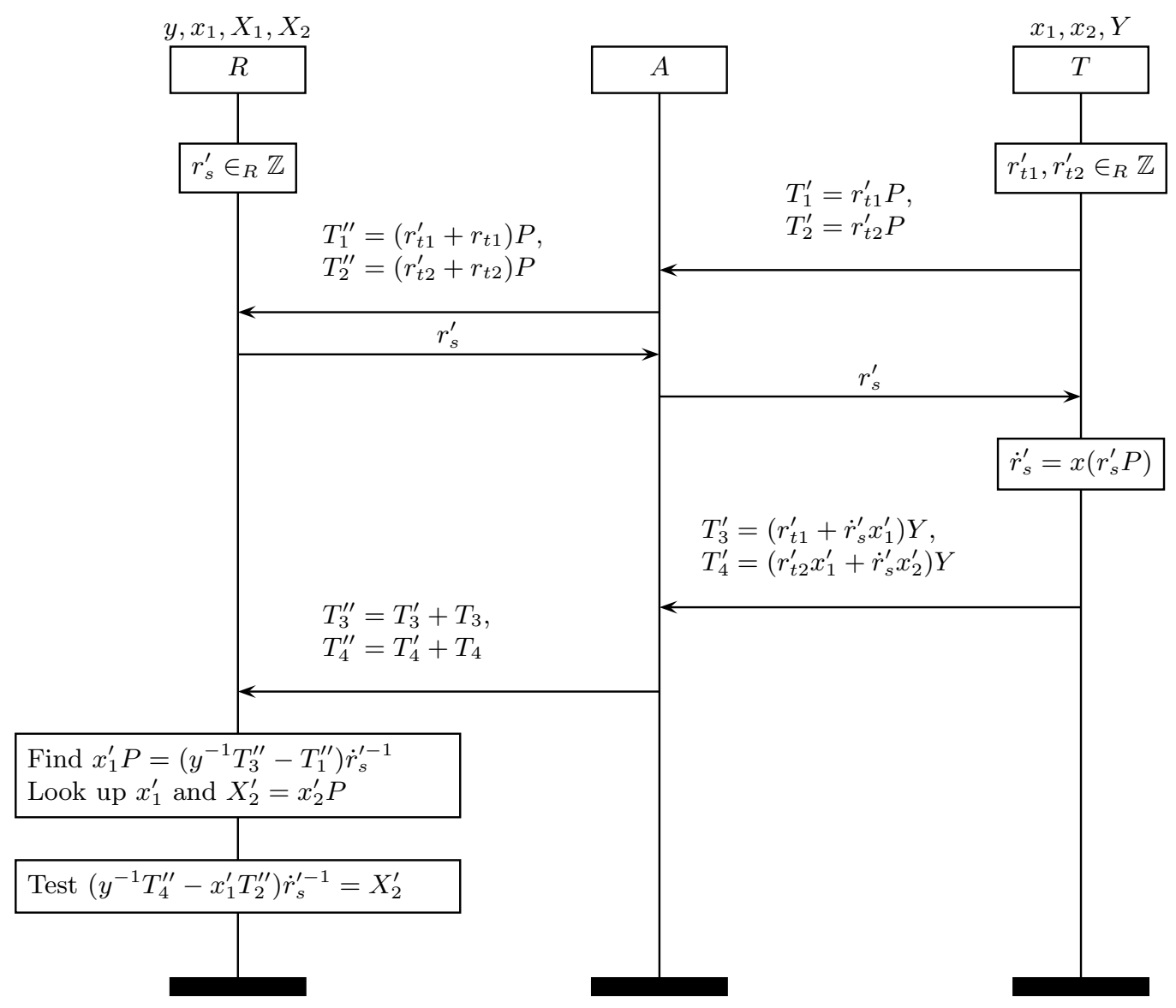

In fact, obtaining such a tuple is trivial by querying the tag twice with the same $r_{s}$ and subtracting the results, since the parts involving $\dot{r}_{s}$ will cancel out. As such we obtain a valid tuple $\left(T_{1}^{*}=r_{t 1}^{*} P, T_{2}^{*}=r_{t 2}^{*} P\right.$, $\left.T_{3}^{*}=r_{t 1}^{*} Y, T_{4}^{*}=r_{t 2}^{*} x_{1} Y\right)$, which can then be used in the first attack. 


\subsection{Third attack}

The third attack shows that the ID-transfer scheme (protocol 1 from [2]) is not wide-strong. A strong attacker is able to read a tag's ID $x_{1}$ without destroying the tag. We will now show how a strong attacker can track a particular tag using a man-in-the-middle attack.

Fig. 6. Man-in-the-middle attack on protocol 1

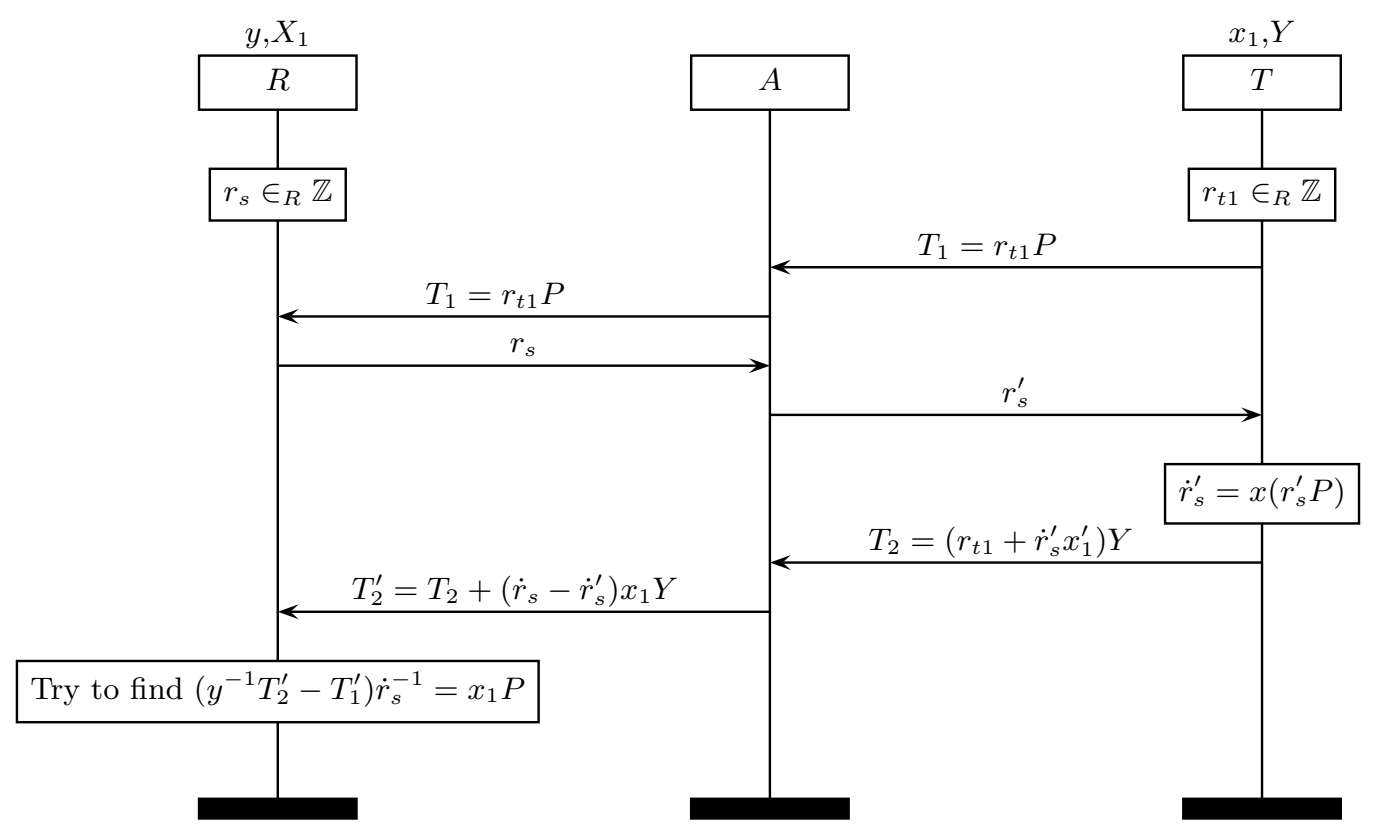

This attack is described in Figure 6. By definition of strong, the attacker knows $x_{1}$ of a certain tag. In order to test if a random tag is the corrupted one, she plays a man-in-the-middle attack as follows. The attacker replaces the value $r_{s}$ with another random value $r_{s}^{\prime}$ and replaces $T_{2}=\left(r_{t 1}+\dot{r}_{s}^{\prime} x_{1}^{\prime}\right) Y$ by

$$
T_{2}^{\prime}=T_{2}+\left(\dot{r}_{s}-\dot{r}_{s}^{\prime}\right) x_{1} Y=\left(r_{t 1}+\dot{r}_{s}^{\prime}\left(x_{1}^{\prime}-x_{1}\right)+\dot{r}_{s} x_{1}\right) Y
$$

The reader will accept this only if $x_{1}=x_{1}^{\prime}$ (provided $\dot{r}_{s}^{\prime} \neq 0$, which the attacker can assure). This allows the attacker to identify the tag $x_{1}$ upon 
acceptance by the reader. The ID-transfer protocol is thus not wide-strong private. Since our attacker is both wide and strong, the ID-transfer might be narrow-strong private or wide-destructive private, although no proof for this is given in the original paper.

\section{Conclusions}

In this paper we have shown three successful attacks on the latest version of EC-RAC [2]. We prove that the ID\&PWD-Transfer scheme is not widestrong private and is not even wide-weak private. The highest possible privacy level that might be achieved by the ID\&PWD-Transfer scheme is narrow-strong privacy.

We also prove that the ID-transfer scheme is not wide-strong private as claimed and can be at most wide-destructive or narrow-strong private.

\section{References}

1. Julien Bringer, Hervé Chabanne, and Thomas Icart. Cryptanalysis of EC-RAC, a RFID identification protocol. In CANS, volume 5339 of Lecture Notes in Computer Science, pages 149-161. Springer, 2008.

2. Yong Ki Lee, Lejla Batina, Dave Singelée, and Ingrid Verbauwhede. Low-Cost Untraceable Authentication Protocols for RFID. In Proceedings of the 3rd ACM conference on Wireless network security (WiSec 2010), Hoboken,NJ,USA, 2010. ACM. Preprint.

3. Yong Ki Lee, Lejla Batina, and Ingrid Verbauwhede. EC-RAC (ECDLP Based Randomized Access Control): Provably Secure RFID authentication protocol. In IEEE International Conference on RFID 2008, pages 97-104, Las Vegas,NA,USA, 2008. IEEE.

4. Yong Ki Lee, Lejla Batina, and Ingrid Verbauwhede. Untraceable RFID Authentication Protocols: Revision of EC-RAC. In IEEE International Conference on RFID 2009, pages 178-185, Orlando,FL,USA, 2009. IEEE.

5. Tatsuaki Okamoto. Provably secure and practical identification schemes and corresponding signature schemes. In CRYPTO '92: Proceedings of the 12th Annual International Cryptology Conference on Advances in Cryptology, pages 31-53, London, UK, 1993. Springer-Verlag.

6. Claus P. Schnorr. Efficient identification and signatures for smart cards. In CRYPTO '89: Proceedings on Advances in cryptology, pages 239-252, New York, NY, USA, 1989. Springer-Verlag New York, Inc.

7. Ton van Deursen and Sasa Radomirovic. Attacks on RFID protocols. Cryptology ePrint Archive, Report 2008/310, 2008. http://eprint.iacr.org/.

8. Ton van Deursen and Sasa Radomirovic. Untraceable RFID protocols are not trivially composable: Attacks on the revision of EC-RAC. Cryptology ePrint Archive, Report 2009/332, 2009. http://eprint.iacr.org/.

9. Serge Vaudenay. On privacy models for RFID. In ASIACRYPT, pages 68-87, 2007. 